\title{
Water Cherenkov Detector installation by the Vertical Muon Equivalent technique
}

\author{
Iskya García* \\ UNIVERSIDAD CENTRAL DE VENEZUELA and UNIVERSIDAD SIMÓN BOLÍVAR, \\ VENEZUELA \\ E-mail: giskya@gmail.com
}

Haydn Barros

UNIVERSIDAD SIMÓN BOLÍVAR, VENEZUELA

E-mail: lab.nuclearegmail.com

José López

UNIVERSIDAD CENTRAL DE VENEZUELA, VENEZUELA

E-mail: jal.ccs@gmail.com

\begin{abstract}
The Large Aperture GRB Obsevatory (LAGO) is a project of The Pierre Auger Observatory that aims the detection of GRB at high altitude sites by the single particle technique (SPT), up today the sites are located in Mexico, Bolivia, Peru, Venezuela and Colombia will join soon. LAGO operates Water Cherenkov Detectors (WCD) arrays whereby is possible to detect the background rates of particles. In Venezuela, there are two collaboration groups: the Universidad de Los Andes and the Universidad Simón Bolívar. The last one is currently installing a prototype of WCD, in this work we present its energy calibration by the Vertical Muon Equivalent (VEM) technique.
\end{abstract}

4th School on Cosmic Rays and Astrophysics

August 25 - September 042010

UFABC - Santo André - São Paulo - Brazil

\footnotetext{
* Speaker.
} 


\section{Introduction}

Gamma-Ray Bursts are flashes of gamma rays associated with extremely energetic explosions in distant galaxies. They are the most luminous events known to occur in the universe.

They are detected at an average rate of a few events per day, and their duration shows a bimodal distribution with two different populations. The short duration GRBs (sGRB), are characterized by durations of less than two seconds, usually thought to be generated by the gravitational coalescence of two compact objects (neutron stars or black holes). On the other hand, long duration GRBs (IGRB) with a duration longer than two seconds, generally associated with the core collapse (collapsar) of massive stars.

The initial burst is usually followed by a longer-lived "afterglow" emitted at longer wavelengths (X-ray, ultraviolet, optical, infrared and radio) [1].

The Large Aperture GRBs Observatory project aims at observing GRBs by the single particle technique using water Cherenkov detectors (WCD). The observatory sites are selected in term of their altitude which is related with the high efficiency to GRB detection:

- Mount Chacaltaya (Bolivia) at 5300 m.a.s.1

- Pico Espejo (Venezuela) at 4765 m.a.s.1

- Sierra Negra (Mexico) at 4600 m.a.s.1

- Peru and Colombia (under investigation)

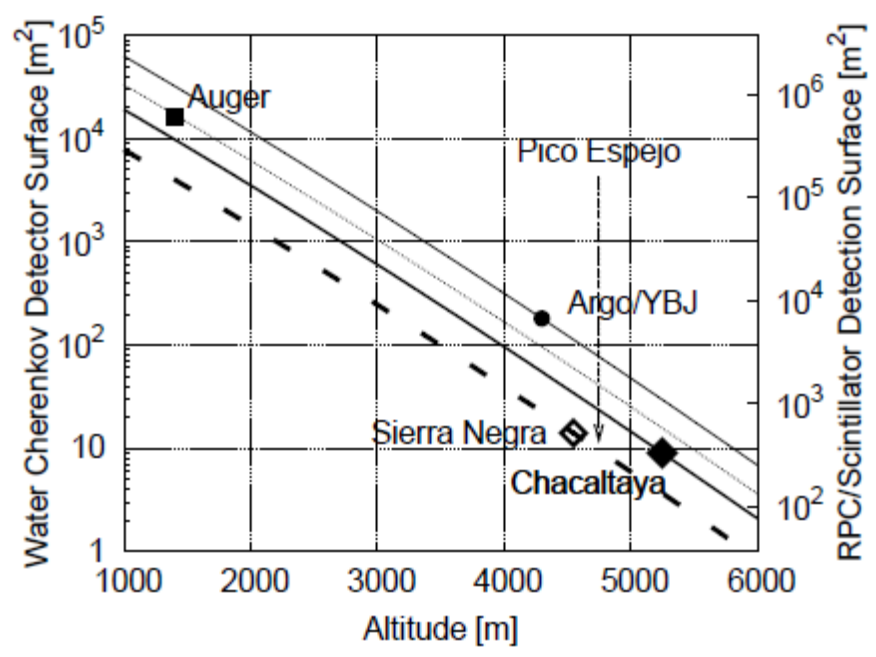

Figure 1: Lines of equal sensitivity for experiments of different size and altitude, neglecting geolatitude cutoff and assuming similar scaler threshold. A few tens of $\mathrm{m}^{2}$ of WCD at high altitude are as efficient as currently running experiments for the SPT (ARGO) [3].

Muons can pass through the tank in various directions with various path lengths. The resulting spectrum has a part originated from low energy particles or short tracks and a prominent maximum due to muons penetrating the tank from the top to the bottom. The characteristics of this maximum (i.e. position and height) correspond to what is called Vertical Equivalent Muon signal [4]. 


\section{Installation of a WCD}

The USB's (Universidad Simón Bolívar) WCD Prototype is a polyetilene tank of 6000 Lts of capacity, its has an exterior cover made of aluminium and asphalt coating, for a good ambient light blocking. Inside the tank, the walls are lining with Tyvek(c), a diffusive reflective material to improve the cherenkov light detection; the tank is filled with water filtered twice, and chemical additive were used to avoid the grow of microorganisms.

It has a PMT of 5", attach to the tank top, which photo sensitive half is placed underwater to detect the cherenkov light produced by charged particles traveling inside the tank.

For the adquisition and processing of data, it was used Nuclear Instrumentation Modules. An amplifier for the output signal of the PMT and an ADC for converting the output signal into a digital signal that can be stored in a PC. The esquemathic representation of the WCD setup could be seen in Figure 2.

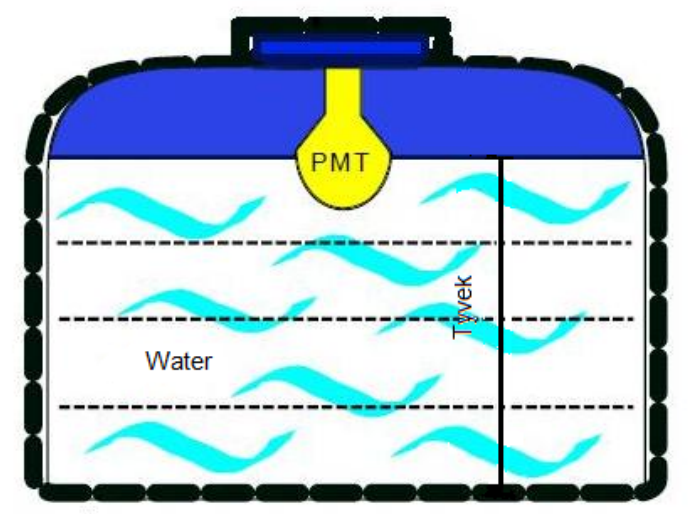

Figure 2: Squemathic representation of WCD

\section{Coincidence Experiment}

For this purpose, it was used a 30x30x4 $\mathrm{cm}^{3}$ plastic scintillator detector (Figure 3), placed under the center of the tank, in order to detect only the muons that cross vertically the tank.

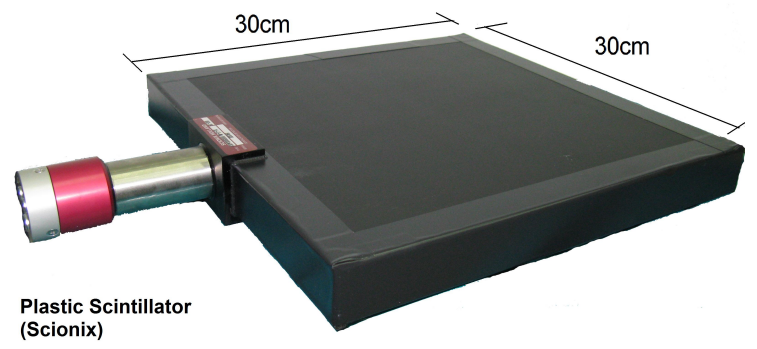

Figure 3: Plastic scintillator detector 
In this case it was used an additional amplifier for the output signal of the scintillator, a coincidence module and the same ADC to digitalize the signal.

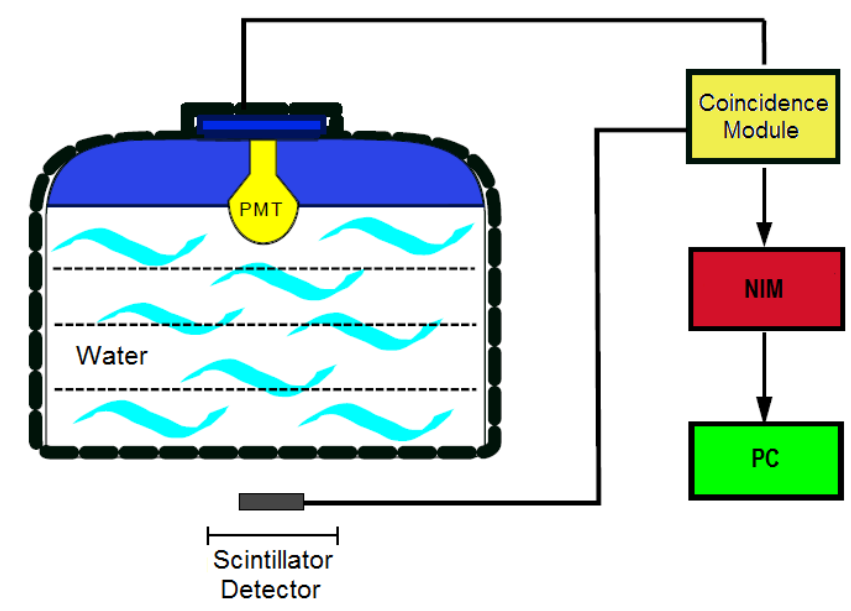

Figure 4: Squemathic representation of coincidence experiment

The output signal of the WCD and scintillator detector goes to their respective amplifier, then those signals goes to the coincidence module, which make a temporal window when a particle travels across the two detectors with a tiny $\Delta t$. This temporal windows is used to trigger the signal coming from the WCD, in which case is obtained only the spectrum of the particle that cross both detectors. The squemathic representation of this setup could be seen in the Figure 4.

This allows to calibrate the WCD in energy, because the energy deposited by the muons along his travel in the WCD can be calculated by Monte Carlo simulations.

\section{Results and Analysis}

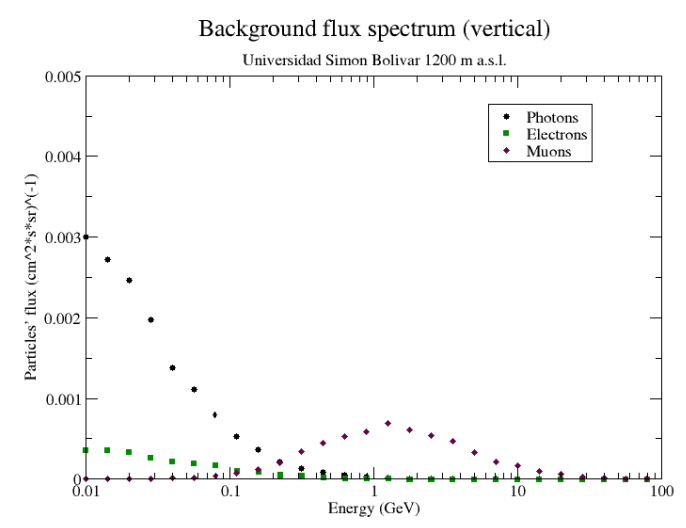

Figure 5: Background flux simulation

The Figure 5 represent the background flux simulation (calculated by means of the software Aires) at 1200 m.a.s.l (USB altitude) in function of the energy, with electromagnetic and hadronic 
component. It simulates usual cosmic ray background $\left(1,8 \times 10^{-2,7} \frac{\text { particles }}{{\mathrm{GeV} * \mathrm{~cm}^{2} * s * s r}^{2}}, 80 \%\right.$ protons and $20 \%$ alfas [2]) arriving from vertical direction.

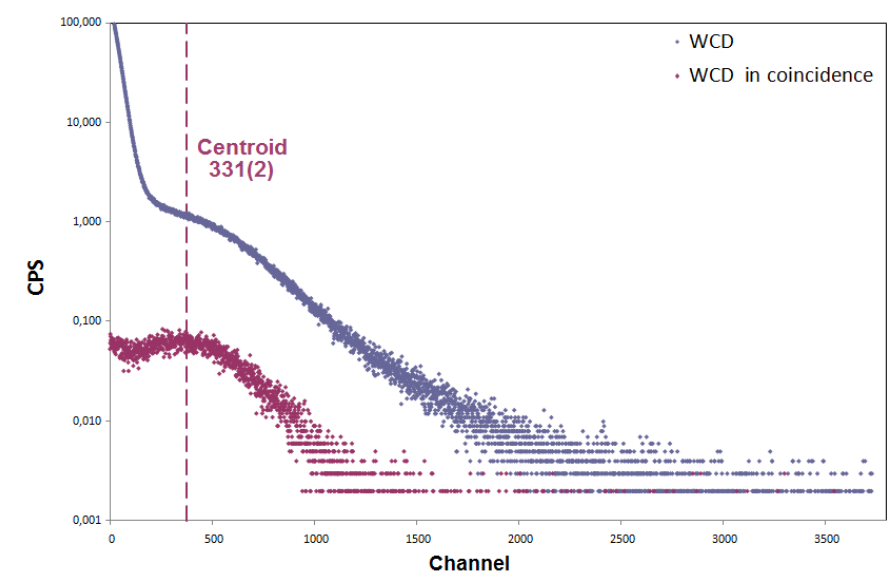

Figure 6: Histogram of charge of the WCD and coincidence experiment

The Figure 6 shows a histogram of charge obtained by the WCD's PMT (blue plot), where the change of the slope represents the muonic component. Its coincidence with the plastic scintillator (purple plot), was gaussian adjusted to determinate the corresponding centroid that represents the VEM channel.

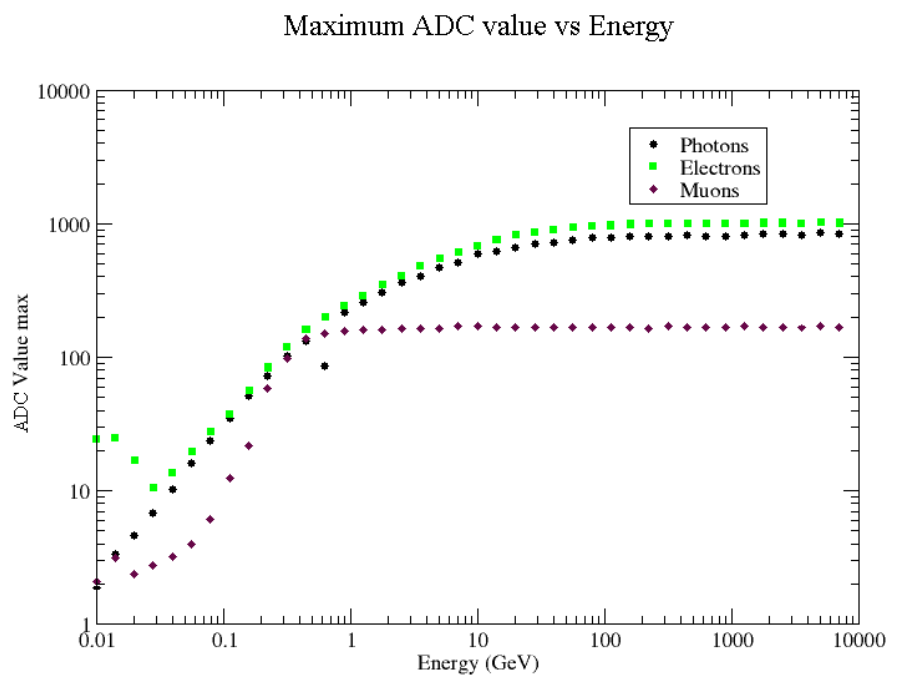

Figure 7: Simulation of the USB's WCD response to a particle's background flux

The Figure 7 correspond to the response of the USB's WCD (1,2 $\mathrm{m}$ of water level), in the case where photons, electrons and muons has a vertical incidence. The plot's vertical axis represent the electrical charge deposited by the detected events, this axis can be affected by a scale fator because the instrumentation: HV, detection area, PMT's quantum eficiency, reflection of Cherenkov light inside WCD, etc. However, this isn't represent a significant change in the simulation because this is only indicative of the order magnitude of the detected events. Instead, the horizontal axis 
represents the deposited energy of the events and it depends principally of the WCD geometry, more especifically of the particle's track length trough the water.

To estimate the energy deposited by a muon that cross vertically (VEM) the WCD, it was used the response simulation of the USB's WCD (Figure 7), where can be seen that at a specific given energy it ocurrs a signal saturation of the plot, in the muon case its because finit limits of the detector(the muon deposited energy is proportional to the track lenght in the WCD). Therefore, the muon plot ( $\mathrm{x} \log$ scale) was adjusted with a sigmoidal function and the point where the saturation ocurrs (99\% of the asimptotic value) was determinated, then the VEM corresponds to a range energy of $1,3-1,8 \mathrm{GeV}$.

\section{Conclusion}

A Water Cherenkov Detector was installed succesfully in the USB (Venezuela). It was used standard nuclear instrumentation, some electronical devices purpose designed and locally available construction materials.

A coincidence experiment was performed to determine the VEM in order to calibrate, in energy, the WCD. The energy range of the VEM (1.2 meters of depth) was determinated and it corresponds to $1,3-1,8 \mathrm{GeV}$.

\section{Acknowledgments}

We wish to express our thanks to the ICTP for the finnancial support of this publication, to the LAGO Project and the Prof. O. Saavedra (Torino University) for lending parts of the engineering equipment and Formalux Iluminación C.A. and Galea C.A. for the partial finantial support of this work.

\section{References}

[1] D. Allard et al. The Large Aperture GRB Observatory. PROCEEDINGS OF THE 31st ICRC, 2009.

[2] K. Nakamura et al.JPG 37 (075021) [http: / / pdg . lbl . gov]. 2010

[3] D. Allard et al. Operating Water Cherenkov Detectors in high altitude sites for the Large Aperture GRB Observatory. PROCEEDINGS OF THE 31st ICRC, 2009.

[4] J. Ridky. The surface detector of the pierre auger observatory. Nuclear Physics B (Proc. Suppl.), 2007. 